Technical Note

\title{
Vibration Analysis of Axially Functionally Graded Non-Prismatic Euler-Bernoulli Beams Using the Finite Difference Method
}

\section{Valentin Fogang}

Civil Engineer, c/o BUNS Sarl, P.O Box 1130, Yaounde, Cameroon; valentin.fogang@bunscameroun.com

ORCID iD https://orcid.org/0000-0003-1256-9862

\begin{abstract}
This paper presents an approach to the vibration analysis of axially functionally graded (AFG) non-prismatic Euler-Bernoulli beams using the finite difference method (FDM). The characteristics (cross-sectional area, moment of inertia, elastic moduli, and mass density) of AFG beams vary along the longitudinal axis. The FDM is an approximate method for solving problems described with differential equations. It does not involve solving differential equations; equations are formulated with values at selected points of the structure. In addition, the boundary conditions and not the governing equations are applied at the beam's ends. In this paper, differential equations were formulated with finite differences, and additional points were introduced at the beam's ends and at positions of discontinuity (supports, hinges, springs, concentrated mass, spring-mass system, etc.). The introduction of additional points allowed us to apply the governing equations at the beam's ends and to satisfy the boundary and continuity conditions. Moreover, grid points with variable spacing were also considered, the grid being uniform within beam segments. Vibration analysis of AFG non-prismatic Euler-Bernoulli beams was conducted with this model, and natural frequencies were determined. Finally, a direct time integration method (DTIM) was presented. The FDM-based DTIM enabled the analysis of forced vibration of AFG non-prismatic Euler-Bernoulli beams, considering the damping. The results obtained in this paper showed good agreement with those of other studies, and the accuracy was always increased through a grid refinement.
\end{abstract}

Keywords: Axially functionally graded non-prismatic Euler-Bernoulli beam; finite difference method; additional points; vibration analysis; direct time integration method

\section{Introduction}

This paper describes the application of Fogang's model [1] based on the finite difference method (FDM), used for the homogeneous Euler-Bernoulli beam, to the vibration analysis of axially functionally graded (AFG) non-prismatic Euler-Bernoulli beam. Various studies have focused on the vibration analysis of AFG Euler-Bernoulli beams, most of which using numerical methods. Chen [2] investigated the bending behavior of a non-uniform AFG Euler-Bernoulli beam based on the Chebyshev collocation method; the Chebyshev differentiation matrices were used to reduce the ordinary differential equations into a set of algebraic equations to form the eigenvalue problem associated with the free vibration. Soltani et al. [3] applied the FDM to evaluate natural frequencies of non-prismatic beams with different 
VIBRATION ANALYSIS OF AXIALLY FUNCTIONALLY GRADED EULER-BERNOULLI BEAMS

boundary conditions and resting on variable one- or two-parameter elastic foundations. Torabi et al. [4] presented an exact closed-form solution for free vibration analysis of Euler-Bernoulli conical and tapered beams carrying any desired number of attached masses; the concentrated masses were modeled by Dirac's delta functions. Liu et al. [5] developed a model for the free transverse vibration of AFG tapered Euler-Bernoulli beam through the spline finite point method; the beam was discretized with a set of uniformly scattered spline nodes along the beam axis, and the displacement field was approximated by the particularly constructed cubic B-spline interpolation functions. Kukla et al. [6] proposed an approach to free vibration analysis of functionally graded beams by approximating the beam by an equivalent beam with piece-wise exponentially varying material and geometrical properties. Cao et al. [7] studied the free vibration of AFG beam using analytical method based on the asymptotic perturbation method and Meijer-Function, respectively.

Classical vibration analysis of Euler-Bernoulli beams involves solving the governing equations that are expressed via means of differential equations, and considering boundary and continuity conditions. However, solving differential equations may be difficult in the presence of an axial force (or external distributed axial forces), an elastic Winkler foundation, a Pasternak foundation, or damping. Generally, the FDM does not consider points outside the beam; the boundary conditions are applied at the beam's ends, not the governing equations. Consequently, the non-application of governing equations at the beam's ends leads to inaccurate results, making the FDM less useful compared with other numerical methods, such as the finite element method. This paper presented a model based on FDM; this model consisted of formulating differential equations with finite differences and introducing additional points at the beam's ends and at positions of discontinuity (supports, hinges, springs, concentrated mass, spring-mass system, etc.). The introduction of additional points allowed us to apply the governing equations at the beam's ends and to satisfy the boundary and continuity conditions. Vibration analysis of structures was conducted using the model, and natural frequencies were determined. Finally, a direct time integration method (DTIM) was presented. The FDM-based DTIM enabled the analysis of forced vibration of Euler-Bernoulli beams, considering the damping.

\section{Materials and methods}

\subsection{Free vibration analysis}

\subsubsection{Governing equations of the free vibration}

The focus here is to determine the eigenfrequencies of the beam. A second-order analysis is conducted; and the first-order analysis can easily be deduced. The sign convention adopted for loads, bending moments, transverse forces, and displacements is illustrated in Figure 1.

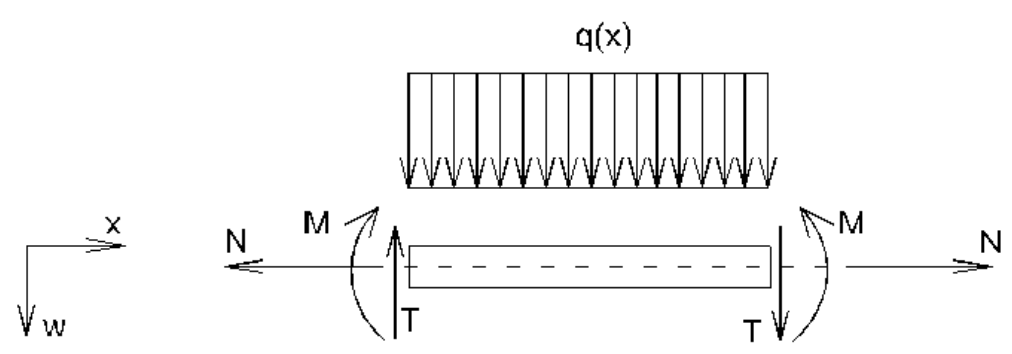

Figure 1 Sign convention for loads, bending moments, transverse forces, and displacements 
Specifically, $\mathrm{M}(x)$ is the bending moment in the section, $\mathrm{T}(x)$ is the transverse force, $\mathrm{N}(x)$ is the axial force (positive in tension), $\mathrm{w}(x)$ is the deflection, and $\mathrm{q}(x)$ is the distributed load in the positive downward direction.

Assuming a harmonic vibration, the equations of dynamic equilibrium on an infinitesimal beam element are as follows:

$$
\begin{aligned}
& \frac{d T(x)}{d x}-k(x) w(x)+\omega^{2} \rho(x) A(x) w(x)=0 \\
& \frac{d M(x)}{d x}+N(x) \frac{d w(x)}{d x}-T(x)=0
\end{aligned}
$$

where $\rho(x)$ is the beam's mass per unit volume, $\mathrm{A}(x)$ is the cross-sectional area, $\mathrm{k}(x)$ is the stiffness of the elastic Winkler foundation, and $\omega$ is the circular frequency of the beam. The transverse force $T(x)$ is related to the shear force $\mathrm{V}(x)$, as follows:

$$
T(x)=V(x)+N(x) \frac{d w(x)}{d x} .
$$

Let us consider an external distributed axial load $\mathrm{n}(x)$ positive along the $+\mathrm{x}$ axis

$$
n(x)=-\frac{d N(x)}{d x}
$$

Substituting Equations (2) and (3) into Equations (1a-b) yields

$$
\begin{aligned}
& \frac{d V(x)}{d x}+N(x) \frac{d^{2} w(x)}{d x^{2}}-n(x) \frac{d w(x)}{d x}-k(x) w(x)+\omega^{2} \rho(x) A(x) w(x)=0 \\
& \frac{d M(x)}{d x}-V(x)=0 .
\end{aligned}
$$

The bending moment, the rotation of the cross section $\varphi(x)$ (positive in clockwise), and the deflection are related together as follows:

$$
M(x)=-E(x) I(x) \frac{d \varphi(x)}{d x} \quad \text { (5a) } \quad \varphi(x)=\frac{d w(x)}{d x}
$$

where at a position $x, \mathrm{E}(x)$ and $\mathrm{I}(x)$ denote the elastic modulus and the moment of inertia, respectively. Combining Equations $(4 a-b)$ and (5a-b) yields

$$
\begin{aligned}
& \frac{d^{2} M(x)}{d x^{2}}+N(x) \frac{d^{2} w(x)}{d x^{2}}-n(x) \frac{d w(x)}{d x}-k(x) w(x)+\omega^{2} \rho(x) A(x) w(x)=0 \\
& M(x)+E(x) I(x) \frac{d^{2} w(x)}{d x^{2}}=0
\end{aligned}
$$


Substituting Equation (6b) into (6a) yields

$-\frac{d^{2}}{d x^{2}}\left[E(x) I(x) \frac{d^{2} w(x)}{d x^{2}}\right]+N(x) \frac{d^{2} w(x)}{d x^{2}}-n(x) \frac{d w(x)}{d x}-k(x) w(x)+\omega^{2} \rho(x) A(x) w(x)=0$.

After some manipulations, Equation (7) yields

$$
\begin{aligned}
& E(x) I(x) \frac{d^{4} w(x)}{d x^{4}}+2 \frac{d[E(x) I(x)]}{d x} \frac{d^{3} w(x)}{d x^{3}}+\left[\frac{d^{2}[E(x) I(x)]}{d x^{2}}-N(x)\right] \frac{d^{2} w(x)}{d x^{2}} \\
& +n(x) \frac{d w(x)}{d x}+\left[k(x)-\omega^{2} \rho(x) A(x)\right] w(x)=0 .
\end{aligned}
$$

Hence, an M-W FDM approximation (Equations (6a-b)) and a W FDM approximation (Equation (8)) can be considered.

\subsubsection{Fundamentals of the FDM}

Let us consider a segment $\mathrm{k}$ of the beam having equidistant grid points with spacing $\mathrm{h}_{\mathrm{k}}$.

Equations (6a-b) have a second-order derivative; consequently, the deflection and bending moment curves $\mathrm{w}(x)$ and $\mathrm{M}(x)$, respectively, are approximated around the point of interest $i$ as second-degree polynomials.

Thus, a three-point stencil is used to write finite difference approximations to derivatives at grid points. The derivatives $(\mathrm{S}(x)$ representing $\mathrm{w}(x)$ or $\varphi(x))$ at $\mathbf{i}$ are expressed with deflection values at points $\mathrm{i}-1, \mathrm{i}$, and $\mathrm{i}+1$.

$$
\left.\frac{d^{2} S(x)}{d x^{2}}\right|_{i}=\frac{S_{i-1}-2 S_{i}+S_{i+1}}{h_{k}^{2}} \quad \text { (9a) }\left.\quad \frac{d S(x)}{d x}\right|_{i}=\frac{-S_{i-1}+S_{i+1}}{2 h_{k}}
$$

Equation (8) has a fourth-order derivative, and the deflection curve is consequently approximated around the point of interest $\mathbf{i}$ as a fourth-degree polynomial. Thus, a five-point stencil is used to write finite difference approximations to derivatives at grid points. The derivatives at $\mathbf{i}$ are expressed with deflection values at points $\mathrm{i}-2, \mathrm{i}-1, \mathrm{i}, \mathrm{i}+1$, and $\mathrm{i}+2$.

$$
\begin{aligned}
& \left.\frac{d^{4} w}{d x^{4}}\right|_{i}=\frac{w_{i-2}-4 w_{i-1}+6 w_{i}-4 w_{i+1}+w_{i+2}}{h_{k}^{4}} \\
& \left.\frac{d^{3} w}{d x^{3}}\right|_{i}=\frac{-w_{i-2}+2 w_{i-1}-2 w_{i+1}+w_{i+2}}{2 h_{k}^{3}} \\
& \left.\frac{d^{2} w}{d x^{2}}\right|_{i}=\frac{-w_{i-2}+16 w_{i-1}-30 w_{i}+16 w_{i+1}-w_{i+2}}{12 h_{k}^{2}} \\
& \left.\frac{d w}{d x}\right|_{i}=\frac{w_{i-2}-8 w_{i-1}+8 w_{i+1}-w_{i+2}}{12 h_{k}}
\end{aligned}
$$




\section{VIBRATION ANALYSIS OF AXIALLY FUNCTIONALLY GRADED EULER-BERNOULLI BEAMS}

\subsubsection{FDM Formulation of equations and efforts}

Two FDM approximations were considered for the analysis of AFG beams: the M-W and the W FDM approximation.

\subsubsection{W FDM approximation}

Since the characteristics of the beam vary throughout the longitudinal axis, reference values are defined. The reference values of the beam's mass per unit volume, the cross-sectional area, the moment of inertia, and the elastic modulus are denoted by $\rho_{\mathrm{r}}, \mathrm{A}_{\mathrm{r}}, \mathrm{I}_{\mathrm{r}}$, and $\mathrm{E}_{\mathrm{r}}$, respectively. At a position $x$ the beam's mass per unit volume, the cross-sectional area, the moment of inertia, the elastic modulus, and the shear modulus are related to the reference values as follows:

$$
\begin{array}{lll}
E(x)=\beta_{E}(x) \times E_{r} & \text { (11a) } & A(x)=\beta_{A}(x) \times A_{r} \\
\rho(x)=\beta_{\rho}(x) \times \rho_{r} & \text { (11b) } & I(x)=\beta_{I}(x) \times I_{r}
\end{array}
$$

The reference length is denoted by $l_{\mathrm{r}}$. We set

$$
h_{k}=\beta_{l k} l_{r} \quad \text { (12a) } \quad W(x)=E_{r} I_{r} \times w(x)
$$

The parameters $\beta^{\prime}{ }_{\mathrm{EI}}(x)$ and $\beta^{\prime \prime}{ }_{\mathrm{EI}}(x)$ are related to the first and second derivative of $\beta_{\mathrm{E}}(x) \beta_{\mathrm{I}}(x)$ with respect to $x$, respectively, as follows:

$$
\beta_{E I}^{\prime}(x)=h_{k} \frac{d\left[\beta_{E}(x) \beta_{I}(x)\right]}{d x}
$$

$$
\beta_{E I}^{\prime \prime}(x)=h_{k}^{2} \frac{d^{2}\left[\beta_{E}(x) \beta_{I}(x)\right]}{d x^{2}}
$$

The vibration frequency $\omega$ is defined as follows:

$$
\omega=\lambda \times \sqrt{\frac{E_{r} I_{r}}{\rho_{r} A_{r} l_{r}^{4}}}
$$

Substituting Equations (10a-d), (11a-d), (12a-b), (13a-b), and (14) into Equation (8) yields the FDM formulation of the governing equation as follows:

$$
\begin{aligned}
& {\left[\beta_{E i} \beta_{I i}-\beta_{E I i}^{\prime}-\frac{\beta_{E I i}^{\prime \prime}}{12}+\frac{N_{i} h_{k}^{2}}{12 E_{r} I_{r}}+\frac{n_{i} h_{k}^{3}}{12 E_{r} I_{r}}\right] W_{i-2}+\left[-4 \beta_{E i} \beta_{I i}+2 \beta_{E I i}^{\prime}+\frac{4 \beta_{E I i}^{\prime \prime}}{3}-\frac{4 N_{i} h_{k}^{2}}{3 E_{r} I_{r}}-\frac{2 n_{i} h_{k}^{3}}{3 E_{r} I_{r}}\right] W_{i-1}} \\
& {\left[6 \beta_{E i} \beta_{I i}-\frac{5 \beta_{E I i}^{\prime \prime}}{2}+\frac{5 N_{i} h_{k}^{2}}{2 E_{r} I_{r}}+\frac{k_{i} h_{k}^{4}}{E_{r} I_{r}}-\beta_{I k}^{4} \beta_{\rho i} \beta_{A i} \lambda^{2}\right] W_{i}+\left[-4 \beta_{E i} \beta_{I i}-2 \beta_{E I i}^{\prime}+\frac{4 \beta_{E I i}^{\prime \prime}}{3}-\frac{4 N_{i} h_{k}^{2}}{3 E_{r} I_{r}}+\frac{2 n_{i} h_{k}^{3}}{3 E_{r} I_{r}}\right] W_{i+1}} \\
& +\left[\beta_{E i} \beta_{I i}+\beta_{E I i}^{\prime}-\frac{\beta_{E I i}^{\prime \prime}}{12}+\frac{N_{i} h_{k}^{2}}{12 E_{r} I_{r}}-\frac{n_{i} h_{k}^{3}}{12 E_{r} I_{r}}\right] W_{i-2}=0
\end{aligned}
$$




\section{VIBRATION ANALYSIS OF AXIALLY FUNCTIONALLY GRADED EULER-BERNOULLI BEAMS}

The bending moment, the shear force, and the rotation of the cross section are calculated using Equations (6b), (10c), (11a), (11d), and (12b), Equations (4b), (6b), (10b-c), (11a), (11d), (12b), and (13a), and Equations (5b), (10d), and (12b) as follows:

$M_{i}=\beta_{E i} \beta_{I i} \frac{W_{i-2}-16 W_{i-1}+30 W_{i}-16 W_{i+1}+W_{i+2}}{12 h_{k}^{2}}$

$V_{i}=\beta_{E I i}^{\prime} \frac{W_{i-2}-16 W_{i-1}+30 W_{i}-16 W_{i+1}+W_{i+2}}{12 h_{k}^{3}}+\beta_{E i} \beta_{I i} \frac{W_{i-2}-2 W_{i-1}+2 W_{i+1}-W_{i+2}}{2 h_{k}^{3}}$

$E_{r} I_{r} \varphi_{i}=\frac{W_{i-2}-8 W_{i-1}+8 W_{i+1}-W_{i+2}}{12 h_{k}}$

Combining Equations (2), (10d), (12b), and (16b) yields the transverse force as follows:

$T_{i}=V_{i}+\frac{N_{i} h_{k}^{2}}{E_{r} I_{r}} \frac{W_{i-2}-8 W_{i-1}+8 W_{i+1}-W_{i+2}}{12 h_{k}^{3}}$

For the special case of a beam without an axial force or a Winkler foundation, Equation (15) becomes

$$
\begin{aligned}
& {\left[\beta_{E i} \beta_{I i}-\beta_{E I i}^{\prime}-\frac{\beta_{E I i}^{\prime \prime}}{12}\right] W_{i-2}+\left[-4 \beta_{E i} \beta_{I i}+2 \beta_{E I i}^{\prime}+\frac{4 \beta_{E I i}^{\prime \prime}}{3}\right] W_{i-1}} \\
& {\left[6 \beta_{E i} \beta_{I i}-\frac{5 \beta_{E I i}^{\prime \prime}}{2}-\beta_{l k}^{4} \beta_{\rho i} \beta_{A i} \lambda^{2}\right] W_{i}+\left[-4 \beta_{E i} \beta_{I i}-2 \beta_{E I i}^{\prime}+\frac{4 \beta_{E I i}^{\prime \prime}}{3}\right] W_{i+1}+\left[\beta_{E i} \beta_{I i}+\beta_{E I i}^{\prime}-\frac{\beta_{E I i}^{\prime \prime}}{12}\right] W_{i-2}=0}
\end{aligned}
$$

\subsubsection{M-W FDM approximation}

Substituting Equations (9a-b), (11a-d), (12a-b), and (14) into Equations (6a-b) yields the FDM formulation of the governing equations as follows:

$$
\begin{aligned}
& h_{k}^{2} M_{i-1}-2 h_{k}^{2} M_{i}+h_{k}^{2} M_{i+1}+\left(\frac{N_{i} h_{k}^{2}}{E_{r} I_{r}}+\frac{n_{i} h_{k}^{3}}{2 E_{r} I_{r}}\right) W_{i-1}-\left(\frac{2 N_{i} h_{k}^{2}}{E_{r} I_{r}}+\frac{k_{i} h_{k}^{4}}{E_{r} I_{r}}-\beta_{l k}^{4} \beta_{A i} \beta_{\rho i} \lambda^{2}\right) W_{i} \\
& +\left(\frac{N_{i} h_{k}^{2}}{E_{r} I_{r}}-\frac{n_{i} h_{k}^{3}}{2 E_{r} I_{r}}\right) W_{i+1}=0 \\
& h_{k}^{2} M_{i}+\beta_{E i} \beta_{I i} W_{i-1}-2 \beta_{E i} \beta_{I i} W_{i}+\beta_{E i} \beta_{I i} W_{i+1}=0
\end{aligned}
$$


The shear force and the rotation of the cross section are calculated using Equations (4b) and (9b), and Equations (5b), (9b), and (12b), respectively, as follows:

$$
V_{i}=\frac{-M_{i-1}+M_{i+1}}{2 h_{k}} \quad \text { (20a) } \quad E_{r} I_{r} \varphi_{i}=\frac{-W_{i-1}+W_{i+1}}{2 h_{k}}
$$

Combining Equations (2), (9b), and (20a) yields the transverse force as follows:

$$
T_{i}=\frac{-M_{i-1}+M_{i+1}}{2 h_{k}}+\frac{N_{i} h_{k}^{2}}{E_{r} I_{r}} \frac{-W_{i-1}+W_{i+2}}{2 h_{k}^{3}}
$$

\subsubsection{Analysis at beam's ends and at positions of discontinuity}

Positions of discontinuity are positions of concentrated mass, spring-mass system, supports, hinges, springs, abrupt change in cross section, positions where $\mathrm{E}(x) \mathrm{I}(x)$ is not differentiable, and change in grid spacing.

The model used in this paper (developed in Fogang [1]) consists of realizing an opening of the beam at the position of discontinuity and introducing additional points in the opening and at beam's ends. Thus, governing equations are applied at the beam's ends, as well as boundary conditions.

In case of the W FDM approximation, additional points (ia, ib, ic, and id) are introduced in the opening, and at beam's ends $(-1,0, n+2$, and $n+3)$, as represented in Figure 2. In case of the M-W FDM approximation, imaginary points (ia and id) are introduced in the opening, and at beam's ends $(0$ and $n+2)$, as represented in Figure 2.

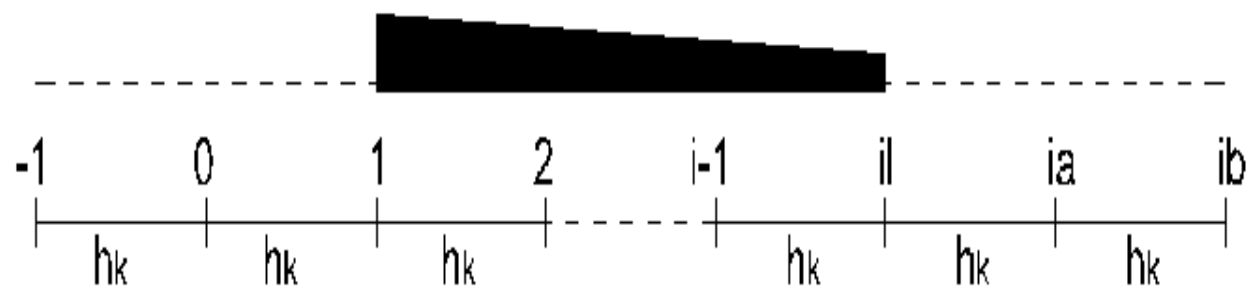

(b)

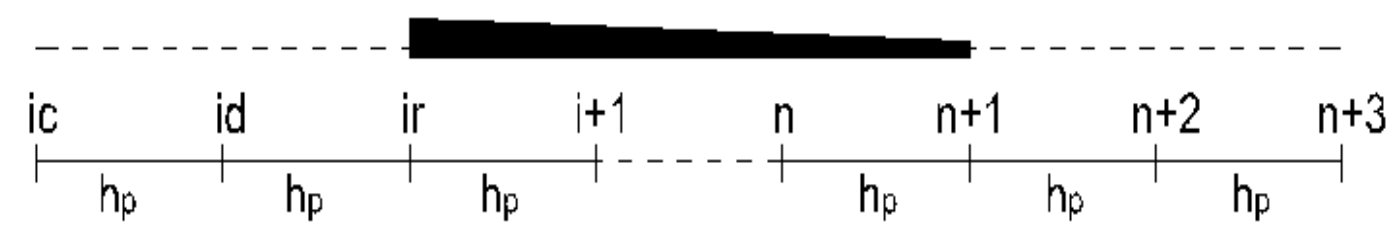

Figure 2 Introduction of imaginary points at beam's ends and in the opening at the left side (a) and right side (b)

Thus, Equation (15) and Equations (19a-b) for W FDM and M-W FDM approximations, respectively, are applied at any point on the grid $(1,2, \ldots \mathrm{i}-1, \mathrm{il}, \mathrm{ir}, \mathrm{i}+1 \ldots \mathrm{n}+1$ of Figure 2$)$. The continuity equations express the continuity of the deflection and rotation of the cross section, and the equilibrium of bending moments and transverse forces. The continuity equations for deflections, rotations of cross sections, and bending moments, respectively, are defined as follows: 


$$
\begin{aligned}
& w_{i l}=w_{i r} \rightarrow W_{i l}=W_{i r} \\
& \varphi_{i l}=\varphi_{i r} \rightarrow E_{r} I_{r} \varphi_{i l}=E_{r} I_{r} \varphi_{i r} \\
& M_{i l}-M_{i r}=0
\end{aligned}
$$

An adjustment of the continuity equations is made e.g. in the case of a hinge (no continuity of the rotation of the cross section, and $\left.\mathrm{M}_{\mathrm{il}}=\mathrm{M}_{\mathrm{ir}}=0\right)$, a support $\left(\mathrm{W}_{\mathrm{il}}=\mathrm{W}_{\mathrm{ir}}=0\right.$, no balance of transverse forces), or a spring.

In case of W FDM approximation, the bending moment and the rotation of the cross section are calculated using Equations (16a) and (16c), respectively, and in case of M-W FDM approximation the rotation of the cross section is calculated using Equation (20b). The balance of transverse forces depends on the case of discontinuity. The transverse forces Til and Tir in Equations (23), (25), and (27a) are calculated using Equations (17) and (21) for W FDM and M-W FDM approximations, respectively.

Effect of a concentrated force $P_{i}$ : The balance of vertical forces yields

$$
T_{i l}-T_{i r}-P_{i}=0
$$

Effect of a concentrated mass: The dynamic behavior of a beam carrying a concentrated mass $\mathrm{M}_{\mathrm{p}}$ was analyzed. We set

$$
M_{p}=m_{p} \times \rho_{r} A_{r} l_{r}
$$

Applying Equations (12b), (14), and (24), the balance of vertical forces yields

$$
T_{i l}-T_{i r}-\frac{M_{p} \omega^{2}}{E_{r} I_{r}} W_{i l}=0 \rightarrow T_{i l}-T_{i r}-\frac{m_{p}}{l_{r}^{3}} \lambda^{2} W_{i l}=0
$$

Effect of a spring-mass system: The dynamic behavior of a beam carrying a spring-mass system was analyzed.

The deflection of the mass is denoted by $\mathrm{W}_{\mathrm{iM}}$. The spring stiffness $\mathrm{K}_{\mathrm{p}}$ is defined as follows:

$$
K_{p}=k_{p} \times E_{r} I_{r} / l_{r}^{3}
$$

Applying Equations (12b), (14), (24), and (26), the balance of vertical forces yields

$$
\begin{aligned}
& T_{i l}-T_{i r}-\frac{M_{p} \omega^{2}}{E_{r} I_{r}} W_{i M}=0 \rightarrow T_{i l}-T_{i r}-\frac{m_{p}}{l_{r}^{3}} \lambda^{2} W_{i M}=0 \\
& \frac{M_{p} \omega^{2}}{E_{r} I_{r}} W_{i M}=\frac{K_{p}}{E_{r} I_{r}} \times\left(W_{i M}-W_{i r}\right) \rightarrow m_{p} \lambda^{2} W_{i M}=k_{p}\left(W_{i M}-W_{i r}\right)
\end{aligned}
$$




\subsection{Direct time integration method}

The direct time integration method used here (developed in Fogang [1]) describes the dynamic response of a beam as a multi-degree-of-freedom system. Viscosity $\eta$ and external loading $\mathrm{p}(x, t)$ are considered. The W FDM approximation is developed in this study; however the M-W FDM approximation can also be considered using appropriate formulas. The equations of dynamic equilibrium on an infinitesimal beam element are as follows:

$$
\begin{aligned}
& E(x) I(x) \frac{\partial^{4} w^{*}(x, t)}{\partial x^{4}}+2 \frac{d[E(x) I(x)]}{d x} \frac{\partial^{3} w^{*}(x, t)}{\partial x^{3}}+\left[\frac{d^{2}[E(x) I(x)]}{d x^{2}}-N(x)\right] \frac{\partial^{2} w^{*}(x, t)}{\partial x^{2}}+ \\
& n(x) \frac{\partial w^{*}(x, t)}{\partial x}+k(x) w^{*}(x, t)=p(x, t)-\rho(x) A(x) \frac{\partial^{2} w^{*}(x, t)}{\partial t^{2}}-\eta \frac{\partial w^{*}(x, t)}{\partial t}
\end{aligned}
$$

The derivatives with respect to $x$ are formulated using Equations (10a-d), while those with respect to $t$ (time increment is $\Delta \mathrm{t})$ are formulated considering a three-point stencil with Equations (29a-c):

$$
\left.\frac{\partial w^{*}(x, t)}{\partial t}\right|_{i, t}=\left.\frac{-w_{i, t-\Delta t}^{*}+w_{i, t+\Delta t}^{*}}{2 \Delta t} \quad \frac{\partial^{2} w^{*}(x, t)}{\partial t^{2}}\right|_{i, t}=\frac{w_{i, t-\Delta t}^{*}-2 w_{i, t}^{*}+w_{i, t+\Delta t}^{*}}{\Delta t^{2}}
$$

At initial time $\mathrm{t}=0$, a three-point forward difference approximation is applied:

$$
\left.\frac{\partial^{2} w^{*}}{\partial t^{2}}\right|_{i, 0}=\left.\frac{w_{i, 0}^{*}-2 w_{i, \Delta t}^{*}+w_{i, 2 \Delta t}^{*}}{\Delta t^{2}} \quad \frac{\partial w^{*}}{\partial t}\right|_{i, 0}=\frac{-3 w_{i, 0}^{*}+4 w_{i, \Delta t}^{*}-w_{i, 2 \Delta t}^{*}}{2 \Delta t}
$$

At final time $\mathrm{t}=\mathrm{T}$, a three-point backward difference approximation is applied:

$$
\left.\frac{\partial^{2} w^{*}}{\partial t^{2}}\right|_{i, T}=\left.\frac{w_{i, T-2 \Delta t}^{*}-2 w_{i, T-\Delta t}^{*}+w_{i, T}^{*}}{\Delta t^{2}} \quad \frac{\partial w^{*}}{\partial t}\right|_{i, T}=\frac{w_{i, T-2 \Delta t}^{*}-4 w_{i, T-\Delta t}^{*}+3 w_{i, T}^{*}}{2 \Delta t}
$$

The governing equation (Equation (28)) can be formulated with the FDM for $\mathrm{x}=\mathrm{i}$ at time $\mathrm{t}$. The FDM formulation of this equation is applied at any point of the beam at any time $t$ using a seven-point stencil. Additional points are introduced to satisfy the boundary and continuity conditions. The boundary conditions are satisfied using a five-point stencil. Thus, beam deflection $\mathrm{w}^{*}(x, t)$ can be determined with the Cartesian model represented in Figure 3 . The bending moment $\mathrm{M}^{*}(x, t)$ and transverse force $\mathrm{T}^{*}(x, t)$ are calculated using Equations (16a) and (17), respectively. 


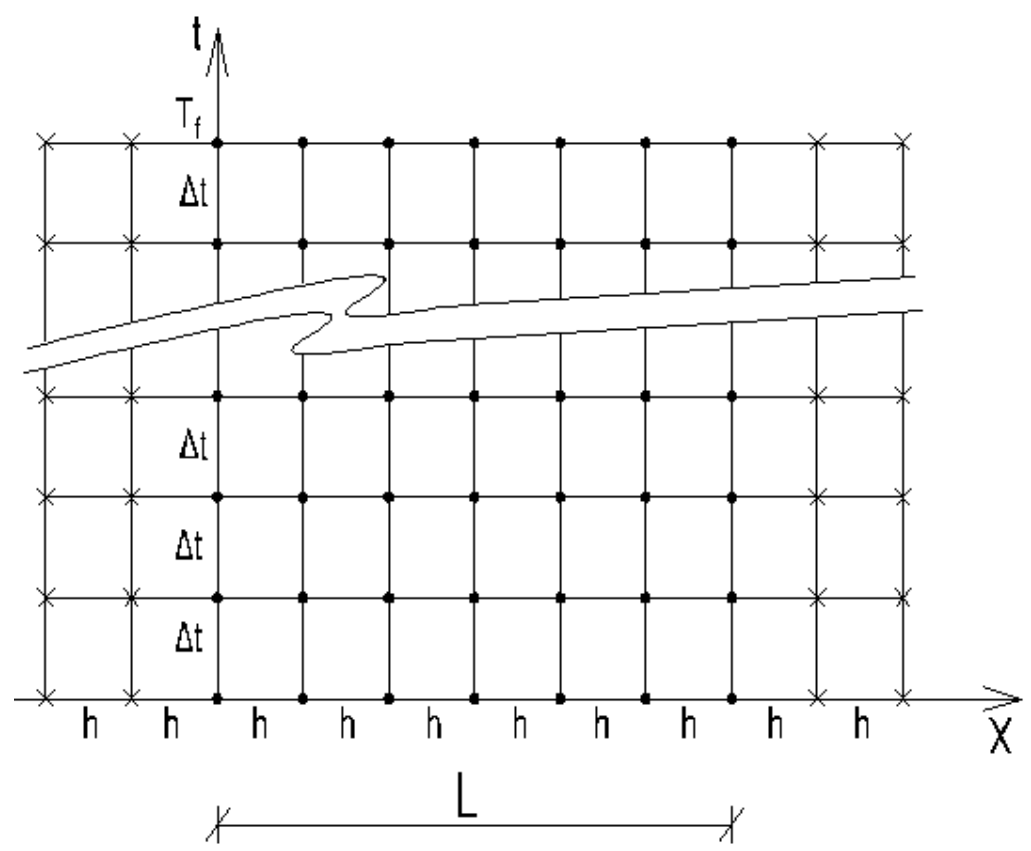

Figure 3 Model for the calculation of time-dependent vibration of axially functionally graded beams

\section{Results and discussion}

\subsection{Free vibration analysis of AFG tapered Euler-Bernoulli beams}

The natural frequencies (coefficients $\lambda$ ) of AFG non-prismatic Euler-Bernoulli beams were determined. Fixed-free, pinned- pinned, and fixed-fixed beams were considered. The geometric and material properties of the beams were represented as follows:

$$
\begin{aligned}
& E(x) I(x)=\left[E_{0}\left(1+\frac{x}{L}\right)\right]\left[\left(1-C_{b} \frac{x}{L}\right)\left(1-C_{h} \frac{x}{L}\right)^{3} I_{0},\right] \\
& \rho(x) A(x)=\left[\rho_{0}\left(1+\frac{x}{L}+\left(\frac{x}{L}\right)^{2}\right)\right]\left[\left(1-C_{b} \frac{x}{L}\right)\left(1-C_{h} \frac{x}{L}\right) A_{0}\right]
\end{aligned}
$$

where $C_{b}$ and $C_{h}$ denote the width and height taper ratios, respectively, and $E_{0}, I_{0}, \rho_{0}$, and $A_{0}$ denote the elastic modulus, the moment of inertia, the beam's mass per unit volume, and the cross-sectional area, respectively, at $\mathrm{x}=0$. FDM Analysis was conducted with $\mathrm{n}=9,17,33$, and 49 grid points for different values of taper ratios and support conditions. Detailed analysis and results are listed in Appendix A and in the Supplementary Materials "Vibration analysis of AFG tapered Euler-Bernoulli beams W FDM approximation" and "Vibration analysis of AFG tapered Euler-Bernoulli beams M-W FDM approximation." The results of this study and those obtained by Chen [2] using the Chebyshev Collocation Method are presented in Table 1. 
Table 1 Coefficients $\lambda$ of natural frequencies (first mode) of AFG Euler-Bernoulli beams: Chen [2], and FDM (W FDM and M-W FDM approximations).

\begin{tabular}{|c|c|c|c|c|c|c|c|c|c|}
\hline \multirow[t]{2}{*}{$\mathrm{C}_{\mathrm{b}} / \mathrm{C}_{\mathbf{h}}$} & \multirow[t]{2}{*}{ Chen [2] } & \multicolumn{2}{|c|}{$\begin{array}{c}\text { FDM } \\
\text { 9-pt grid }\end{array}$} & \multicolumn{2}{|c|}{$\begin{array}{c}\text { FDM } \\
\text { 17-pt grid }\end{array}$} & \multicolumn{2}{|c|}{$\begin{array}{c}\text { FDM } \\
\text { 33-pt grid }\end{array}$} & \multicolumn{2}{|c|}{$\begin{array}{c}\text { FDM } \\
\text { 49-pt grid }\end{array}$} \\
\hline & & $\mathbf{W}$ & $\mathbf{M}-\mathbf{W}$ & $\mathbf{W}$ & M-W & $\mathbf{W}$ & $\mathbf{M}-\mathbf{W}$ & $\mathbf{W}$ & M-W \\
\hline \multicolumn{10}{|c|}{ Fixed-free beam } \\
\hline$\overline{0.0 / 0.0}$ & 2.4256 & 2.4237 & 2.3754 & 2.4250 & 2.4128 & 2.4254 & 2.4223 & 2.4255 & 2.4241 \\
\hline $0.4 / 0.2$ & 2.9336 & 2.8581 & 2.8946 & 2.9141 & 2.9237 & 2.9287 & 2.9311 & 2.9314 & 2.9325 \\
\hline $0.2 / 0.4$ & 2.7987 & 2.67272 & 2.7649 & 2.7658 & 2.7902 & 2.7904 & 2.7966 & 2.7950 & 2.7978 \\
\hline $0.6 / 0.8$ & 3.9232 & 3.3814 & 3.9416 & 3.7598 & 3.9278 & 3.8803 & 3.9244 & 3.9040 & 3.9237 \\
\hline \multicolumn{10}{|c|}{ Pinned-pinned beam } \\
\hline $0.0 / 0.0$ & 9.0286 & 8.9246 & 8.9147 & 9.0024 & 8.9999 & 9.0220 & 9.0214 & 9.0256 & 9.0254 \\
\hline $0.4 / 0.2$ & 8.1498 & 8.0858 & 8.0449 & 8.1339 & 8.1234 & 8.1458 & 8.1432 & 8.1480 & 8.1469 \\
\hline $0.2 / 0.4$ & 7.1455 & 7.0821 & 7.0526 & 7.1294 & 7.1221 & 7.1415 & 7.1397 & 7.1437 & 7.1429 \\
\hline $0.6 / 0.8$ & 4.4263 & 4.0988 & 4.3874 & 4.6027 & 4.4136 & 4.4234 & 4.4229 & 4.4243 & 4.4247 \\
\hline \multicolumn{10}{|c|}{ Fixed-fixed } \\
\hline$\overline{0.0 / 0.0}$ & 20.4721 & 20.1752 & 19.2218 & 20.3980 & 20.1402 & 20.4550 & 20.3878 & 20.4638 & 20.4345 \\
\hline $0.4 / 0.2$ & 18.1286 & 17.9344 & 16.9614 & 18.0798 & 17.8178 & 18.1163 & 8.0495 & 18.1231 & 18.0933 \\
\hline $0.2 / 0.4$ & 15.8497 & 15.6817 & 14.7663 & 15.8031 & 15.5586 & 15.8377 & 5.7755 & 15.8444 & 15.8166 \\
\hline $0.6 / 0.8$ & 10.5143 & 7.1533 & 9.4032 & 12.4511 & 10.1105 & 10.4699 & 0.4002 & 10.4895 & 10.4623 \\
\hline
\end{tabular}

The results of this study are in good agreement with those of Chen [2].

\section{Conclusion}

The FDM-based model developed in this paper enabled, with relative easiness, vibration analysis of axially functionally graded non-prismatic Euler-Bernoulli beams. The results showed that the calculations conducted as described in this paper were accurate.

The following aspects were not addressed in this study but could be analyzed with the model in the future:

Axially functionally graded Euler-Bernoulli beams resting on Pasternak foundations

Elastically connected multiple-beam system

First- and second-order dynamic stiffness matrices 
Supplementary Materials: The following files were uploaded during submission:

- "Vibration analysis of AFG tapered Euler-Bernoulli beams W FDM approximation"

- "Vibration analysis of AFG tapered Euler-Bernoulli beams M-W FDM approximation"

\section{Funding:}

\section{Acknowledgments:}

Conflicts of Interest: The author declares no conflict of interest.

\section{Appendix A: Vibration analysis of AFG tapered Euler-Bernoulli beams}

Applying Equations (11a-d), (12a), and (13a-b), the parameters $\beta_{\mathrm{I}}(\mathrm{x}), \beta_{\mathrm{A}}(\mathrm{x}), \beta_{\rho}(\mathrm{x}), \beta_{\mathrm{E}}(\mathrm{x}), \beta_{\mathrm{EI}}^{\prime}(\mathrm{x})$, and $\beta_{\mathrm{EI}}^{\prime \prime}(\mathrm{x})$ are calculated as follows:

$$
\begin{aligned}
& \beta_{I}(x)=\left(1-C_{b} \frac{x}{L}\right)\left(1-C_{h} \frac{x}{L}\right)^{3} \quad \beta_{A}(x)=\left(1-C_{b} \frac{x}{L}\right)\left(1-C_{h} \frac{x}{L}\right) \\
& \beta_{E}(x)=1+\frac{x}{L} \quad \beta_{\rho}(x)=1+\frac{x}{L}+\left(\frac{x}{L}\right)^{2} \\
& \beta_{E I}^{\prime}(x)=\beta_{l k}\left[1-C_{b}-2 C_{b} \frac{x}{L}\right]\left(1-C_{h} \frac{x}{L}\right)^{3}-3 \beta_{l k} C_{h}\left[1+\left(1-C_{b}\right) \frac{x}{L}-C_{b}\left(\frac{x}{L}\right)^{2}\right]\left(1-C_{h} \frac{x}{L}\right)^{2} \\
& \beta_{E I}^{\prime \prime}(x)=-2 \beta_{l k}^{2} C_{b}\left(1-C_{h} \frac{x}{L}\right)^{3}-6 \beta_{l k}^{2} C_{h}\left[1-C_{b}-2 C_{b} \frac{x}{L}\right]\left(1-C_{h} \frac{x}{L}\right)^{2}+ \\
& 6 \beta_{l k}^{2} C_{h}^{2}\left[1+\left(1-C_{b}\right) \frac{x}{L}-C_{b}\left(\frac{x}{L}\right)^{2}\right]\left(1-C_{h} \frac{x}{L}\right)
\end{aligned}
$$

\section{References}

[1] Fogang, V. Euler-Bernoulli Beam Theory: First-Order Analysis, Second-Order Analysis, Stability, and Vibration Analysis Using the Finite Difference Method. Preprints 2021, 2021020559 (doi: 10.20944/preprints202102.0559.v2).

[2] Chen, W.R. Vibration Analysis of Axially Functionally Graded Tapered Euler-Bernoulli Beams Based on Chebyshev Collocation Method. International Journal of Acoustics and Vibration, Vol. 25, No. 3, 2020. https://doi.org/10.20855/ijav.2020.25.31680

[3] M. Soltani, A. Sistani, B. Asgarian. Free Vibration Analysis of Beams with Variable Flexural Rigidity Resting on one or two Parameter Elastic Foundations using Finite Difference Method. Conference Paper. The 2016 World Congress on The 2016 Structures Congress (Structures16) 
[4] K. Torabi, H. Afshari, M. Sadeghi, H. Toghian. Exact Closed-Form Solution for Vibration Analysis of Truncated Conical and Tapered Beams Carrying Multiple Concentrated Masses. Journal of Solid Mechanics Vol. 9, No. 4 (2017) pp. 760-782

[5] P. Liu, K. Lin, H. Liu, R. Qin. Free Transverse Vibration Analysis of Axially Functionally Graded Tapered EulerBernoulli Beams through Spline Finite Point Method. Shock and Vibration Volume 2016, Article ID 5891030, 23 pages. http://dx.doi.org/10.1155/2016/5891030

[6] S. Kukla, J. Rychlewska. An Approach to Free Vibration Analysis of Axially Graded Beams. Journal of Theoretical and Applied Mechanics 54, 3, pp. 859-870, Warsaw 2016. DOI: 10.15632/jtam-pl.54.3.859

[7] Dongxing Cao, Bin Wang, Wenhua Hu and Yanhui Gao (April 22nd 2019). Free Vibration of Axially Functionally Graded Beam, Mechanics of Functionally Graded Materials and Structures, Farzad Ebrahimi, IntechOpen, DOI: 10.5772/intechopen.85835. Available from: https://www.intechopen.com/books/mechanics-offunctionally-graded-materials-and-structures/free-vibration-of-axially-functionally-graded-beam 